\title{
NUEVAS ESPECIES Y CITAS IBÉRICAS DE CTENOBELBA S. STR. BALOGH, 1943, Y DESCRIPCIÓN DE CTENOBELBA (AOKIBELBA) N. SUBG. DEL ESTE DE ASIA (ACARI, ORIBATIDA, CTENOBELBIDAE)
}

\author{
L. S. Subías ${ }^{1} \&$ U. Ya. Shtanchaeva ${ }^{2}$
}

\section{RESUMEN}

L. S. Subías \& U. Ya. Shtanchaeva. 2013. Nuevas especies y citas ibéricas de Ctenobelba s. str. Balogh, 1943, y descripción de Ctenobelba (Aokibelba) n. subg. del este de Asia (Acari, Oribatida, Ctenobelbidae). Grael/sia, 69(1): 37-44.

Se realizan una serie de consideraciones generales sobre el género Ctenobelba Balogh, 1943, y se describe un nuevo subgénero, Ctenobelba (Aokibelba) n. subg., para las especies asiáticas. Se describen además tres nuevas especies de la Península Ibérica: Ctenobelba (C.) pseudomahnerti n. sp., Ctenobelba (C.) parapulchellula n. sp. y Ctenobelba (C.) foveolata n. sp. Se citan también por primera vez para la Península lbérica las especies: Ctenobelba (C.) brevipilosa Mahunka, 1964, Ctenobelba (C.) marcuzzii Mahunka, 1974 y Ctenobelba (C.) csiszarae Mahunka, 1977, y se ofrece una clave de identificación de las especies ibéricas del subgénero Ctenobelba s. str.

Palabras clave: Ácaros; Oribátidos; Ctenobelbidae; Nuevo subgénero; Nuevas especies; Nuevas citas; Claves de especies; Península Ibérica

\section{ABSTRACT}

L. S. Subías \& U. Ya. Shtanchaeva. 2013. New species and new lberian records of Ctenobelba s. str. Balogh, 1943, and description of Ctenobelba (Aokibelba) n. subg. from East Asia (Acari, Oribatida, Ctenobelbidae). Graellsia, 69(1): 37-44 (in Spanish).

Some general observations about the genus Ctenobelba Balogh, 1943 are made. The new subgenus Ctenobelba (Aokibelba) n. subg. is created to include Asiatic species. Three new species from the Iberian Peninsula: Ctenobelba (C.) pseudomahnerti n. sp., Ctenobelba (C.) parapulchellula n. sp. and Ctenobelba (C.) foveolata n. sp. are described. Also the species: Ctenobelba (C.) brevipilosa Mahunka, 1964, Ctenobelba (C.) marcuzzii Mahunka, 1974 and Ctenobelba (C.) csiszarae Mahunka, 1977 are recorded for the first time from the Iberian Peninsula, and a key to the Iberian species of the subgenus Ctenobelba s. str. is given.

Key words: Oribatid mites; Ctenobelbidae; New subgenus; New species; New records; Key of species; Iberian Peninsula.

\footnotetext{
Departamento de Zoología. Facultad de Biología. Universidad Complutense. 28040 Madrid. España. E-mail: subias@bio.ucm.es

2 Instituto de Recursos Biológicos del Caspio de Daguestán. Academia de Ciencias de Rusia. Mahachkala 376000. Rusia. Email: umukusum@mail.ru
} 


\section{Introducción}

El género Ctenobelba Balogh, 1943 presenta una distribución fundamentalmente Paleártica que se ha introducido en el norte de la Region Oriental, y actualmente incluye 25 especies, 23 dentro del subgénero típico Ctenobelba $s$. str., una española dentro del subgénero Ctenobelba (Bifurcobelba) Subías y Shtanchaeva, 2010, y otra caucásica dentro del subgénero Ctenobelba (Caucasiobelba) Subías y Shtanchaeva, 2010 (Subías, 2004 y 2012). El subgénero Ctenobelba s. str. está presente en la Península Ibérica con 8 especies válidas (Subías \& Shtanchaeva, 2012): Ctenobelba (C.) apatomorpha Iturrondobeitia, Saloña, Andrés y Caballero, 1998, Ctenobelba (C.) foliata Hammer, 1961, Ctenobelba (C.) mahnerti Mahunka, 1974, Ctenobelba (C.) parafoliata Pérez-Íñigo jr., 1991, Ctenobelba (C.) pectinigera (Berlese, 1908), Ctenobelba (C.) perezinigoi Moraza, 1985, Ctenobelba (C.) pilosella Jeleva, 1962 y Ctenobelba (C.) pulchellula GilMartín y Subías, 1997, a las que hay que añadir Ctenobelba (Bifurcobelba) iberica Subías y Shtanchaeva, 2010. En el presente trabajo se va proceder a la creación de un nuevo subgénero de Ctenobelba con las especies asiáticas y a describir tres nuevas especies, así como a realizar tres nuevas citas del subgénero típico, de diversas localidades de España.

\section{Material y métodos}

Todos los ejemplares estudiados proceden de muestras edáficas y han sido extraidos por el método Berlese-Tullgrenn. Se encuentran depositados en la Colección de la Cátedra de Entomología de la Facultad de Biología de la Universidad Complutense de Madrid y conservados bien en tubos con ácido láctico al 70\%, bien en preparaciones microscópicas con "Hoyer".

\section{Resultados}

Ctenobelba Balogh, 1943

ESPECIE TIPO: Eremobelba pectinigera Berlese, 1908

Se trata de un género de características meridionales ya que de las 16 especies europeas válidas, sólo tres han sido citadas de centroeuropa:
Ctenobelba pectinigera (Berlese, 1908) (Weigmann, 2006), Ctenobelba brevipilosa Mahunka, 1964 y Ctenobelba csiszarae Mahunka, 1977, mientras que las demás son mediterráneas. Por lo que respecta a las otras cinco especies del género, están distribuidas por el este de la región Paleártica oriental: Ctenobelba longisetosa Suzuoka y Aoki, 1980 descrita de Japón y encontrada posteriormente en otras localidades del este de la región Paleártica oriental, Ctenobelba soloduchi Pankov, 1988 del este de Rusia asiática, Ctenobelba polysetosa Aoki y Yamamoto, 2000 descrita de China suroriental y encontrada posteriormente en Japón, Ctenobelba leei Choi, 2005 de Corea y Ctenobelba nakatamarii Aoki, 2007 de Japón (Subías, op. cit.). Según Aoki (2007), Choi (2005) divide el género Ctenobelba en dos grupos, el grupo "pectinigera" de especies europeas, con setas notogastrales diminutas o medianas y setas epimerales simples, y el grupo "longisetosa" de especies del Extremo Este de Asia, con setas notogastrales largas o muy largas y setas epimerales ramificadas que incluiría las especies $C$. longiseto$s a, C$. polysetosa y Ctenobelba leei, al que pertenecería su nueva especie Ctenobelba nakatamarii Aoki, 2007. A estas especies debería de añadirse Ctenobelba soloduchi, especie descrita por Pankov (1988) del Extremo Este de Rusia y que no fue considerada por los mencionados autores. Se considera que dichas características distintivas entre ambos grupos, junto con la distribución geográfica tan distinta, son suficiente motivo como para la creación de un nuevo subgénero de Ctenobelba, que se dedica al prestigioso oribatólogo japonés Dr. Jun-ichi Aoki.

Ctenobelba (Aokibelba) n. subg.

ESPECIE TIPO: Ctenobelba longisetosa Suzuoka y Aoki, 1980

\section{Clave de especies de Ctenobelba (Aokibelba)}

1. Sensilo setiforme barbulado

- Sensilo pectinado C. (A.) nakatamarii Aoki, 2007 n. comb.

2. Setas de la placa ventral ramificadas ............................ 3

- Setas de la placa ventral simples .................................... 4

3. Setas genitales ramificadas .. C. (A.) polysetosa Aoki y Yamamoto, 2000 n. comb. - Setas genitales simples C. (A.) soloduchi Pankov, 1988 n. comb. 

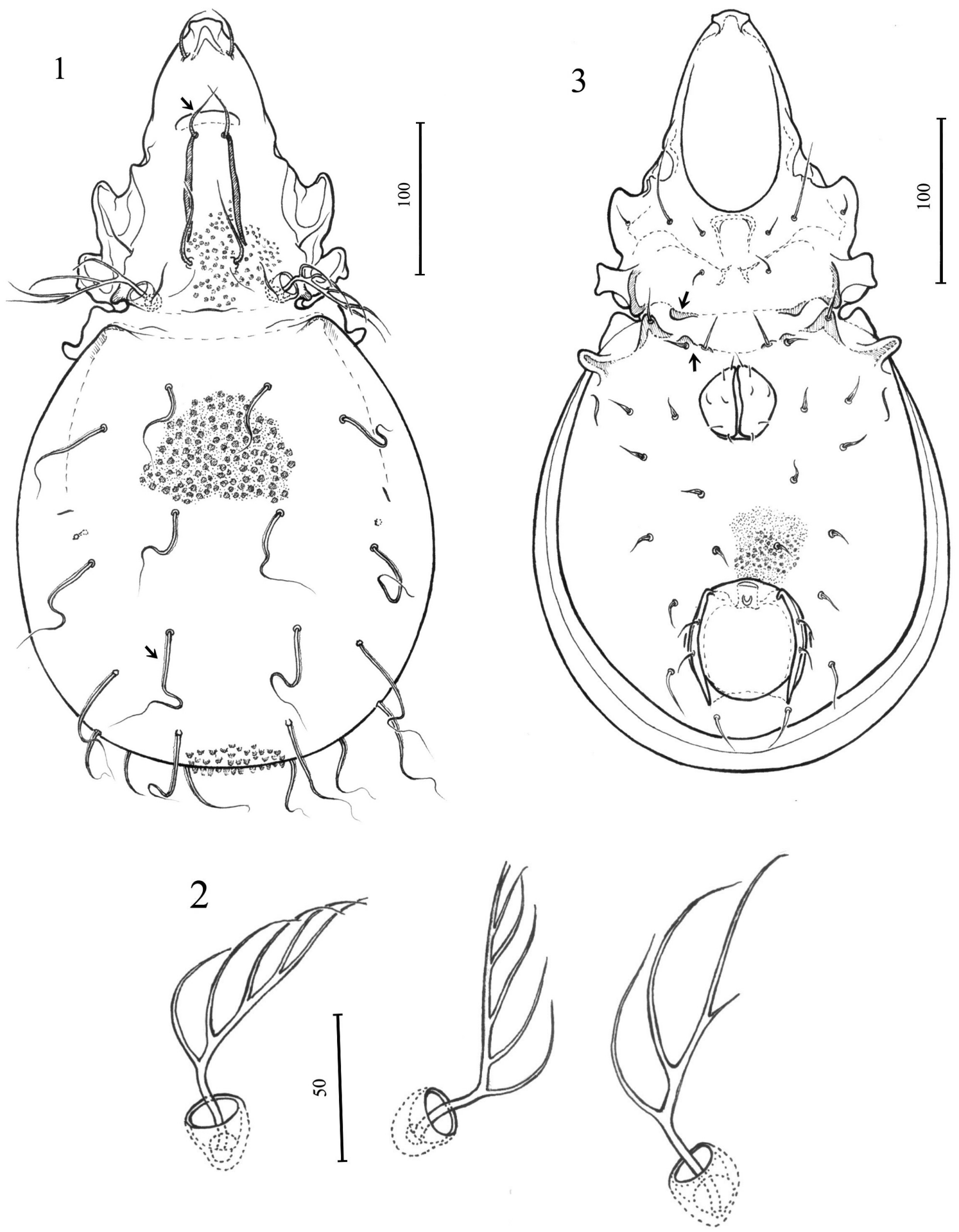

Figs. 1-3.- Ctenobelba (C.) pseudomahnerti n. sp.: 1) Vista dorsal del cuerpo; 2) diferentes tipos de sensilos; 3) vista ventral del cuerpo. (Escala en $\mu \mathrm{m}$ ).

Figs. 1-3.- Ctenobelba (C.) pseudomahnerti n. sp.: 1) Dorsal view of the body; 2) different kind of sensilli; 3) ventral view of the body. (Scale bar in $\mu \mathrm{m})$. 
4. Setas epimerales largas y de fórmula 3-1-3-3 C. (A.) leei Choi, 2005 n. comb.

- Setas epimerales cortas y de fórmula 3-1-3-4 .... C. (A.) longisetosa Suzuoka y Aoki, 1980 n. comb.

\section{Ctenobelba (C.) pseudomahnerti n. sp.}

MATERIAL TIPO: Se han estudiado 27 ejemplares de una muestra del noroeste de España, de Moscoso (Pontevedra) recolectada el 24 de abril de 2011 por R. Outerelo en un talud; el holotipo se encuentra conservado en ácido láctico al $70 \%$ y los 26 paratipos montados en una preparación microscópica con Hoyer.

DESCRIPCIÓN. Dimensiones: sus medidas oscilan entre los 475-525 $\mu \mathrm{m}$ de longitud por $245-270 \mu \mathrm{m}$ de anchura.

Prodorso (Fig. 1): cubierto por un cerotegumento granuloso aciculado densa e irregularmente dispuesto, presenta un rostro prominente con dos dientes romos laterales; las setas rostrales son ásperas y de una longitud similar a la de las setas lamelares, que se insertan en el extremo de las cóstulas lamelares que son largas y paralelas, siendo también patente por delante de ellas una línea translamelar arqueada; las setas interlamelares, también ásperas, son más largas y se disponen cerca de la base de las cóstulas lamelares; los sensilos (Fig. 2) son pectinados y normalmente con 5-6 largas ramas (excepcionalmente 4 ó 7); las setas exobotrídicas son largas pero finas y lisas, siendo de destacar la presencia de sendas apófisis prodorsales prominentes por fuera de los botridios.

Notogáster (Fig. 1): también cubierto por un denso e irregularmente dispuesto granulado aciculado, pero de mayor tamaño que el prodorsal; los bordes humerales son angulosos y el borde anterior notogastral ligeramente cóncavo con el par de apófisis que se sitúan por detrás de los botridios apenas insinuadas; los 10 pares de setas notogastrales son muy largas, lisas y acabadas en un largo extremo flageliforme, y presentan un revestimiento hialino apenas perceptible en su mitad basal; las fisuras im son cortas pero bien patentes.

Región ventral (Fig. 3): se encuentra cubierta por un cerotegumento granuloso similar al prodorsal; la región epimeral presenta dos pares de apófisis sejugales enfrentadas muy desarrolladas, similares a las que representa Iordansky (1999) para la especie Ctenobelba translamellata que describe de Ucrania; las setas epimerales son finas y lisas y en el número habitual (3:1:3:3); las setas $1 b$ son considerablemente más largas que las demás, mientras que las $3 a$ y $3 b$ se disponen sobre sendas pequeñas apófisis sejugales medias posteriores y las $3 c$ sobre las más desarrolladas apófisis sejugales posteriores laterales; los discidios presentan un desarrollo similar al de estas apófisis y el borde epimeral posterior no está desarrollado, y las setas de las serie 4 presentan un reborde hialino basal; los 3 pares de setas adgenitales de la placa ventral son similares a éstas y los 6 pares de setas genitales son diminutos, mientras que los 2 pares de anales y los 3 pares de adanales están más desarrollados; las fisuras adanales iad son pequeñas y paraanales, disponiéndose junto a las placas anales aproximadamente a su altura media; las patas son moniliformes, monodáctilas y no están cubiertas de cerotegumento.

ETIMología. El epíteto "pseudomahnerti" hace referencia a su semejanza con C. mahnerti.

Discusión. A la especie que más se asemeja es a C. mahnerti, pero se diferencia claramente de ella porque el granulado notogastral en la nueva especie está distribuido irregularmente y no en forma reticulada.

\section{Ctenobelba (C.) parapulchellula $\mathbf{n}$. sp.}

MATERIAL TIPO: Se han estudiado 6 ejemplares procedentes del sur de la Península Ibérica, 4 de ellos procedentes del sureste de España, de Parauta (Málaga), recolectada por L. S. Subías el 23 de marzo de 1983 en un pinar (de donde se ha designado el holotipo que se encuentra conservado en ácido láctico al $70 \%$ ), y los otros 2 del sur de Portugal, de Sines, procedentes de una muestra de matorral de dunas recolectada también por L. S. Subías el 2 de marzo de 1983. Todos los paratipos se encuentran montados en preparaciones microscópicas con Hoyer.

DESCRIPCIÓN. Dimensiones: sus medidas oscilan entre los 400-450 $\mu \mathrm{m}$ de longitud por $215-245 \mu \mathrm{m}$ de anchura.

Prodorso (Fig. 4): cubierto por un cerotegumento granuloso aciculado irregularmente dispuesto, como en la especie precedente; el rostro, prominente y con dos dientes laterales, las setas rostrales, lamelares e interlamelares ásperas, las cóstulas lamelares, largas y paralelas, y los sensilos, con 56 largas ramas, son también muy similares a los de la especie precedente, pero las setas exobotrídicas 


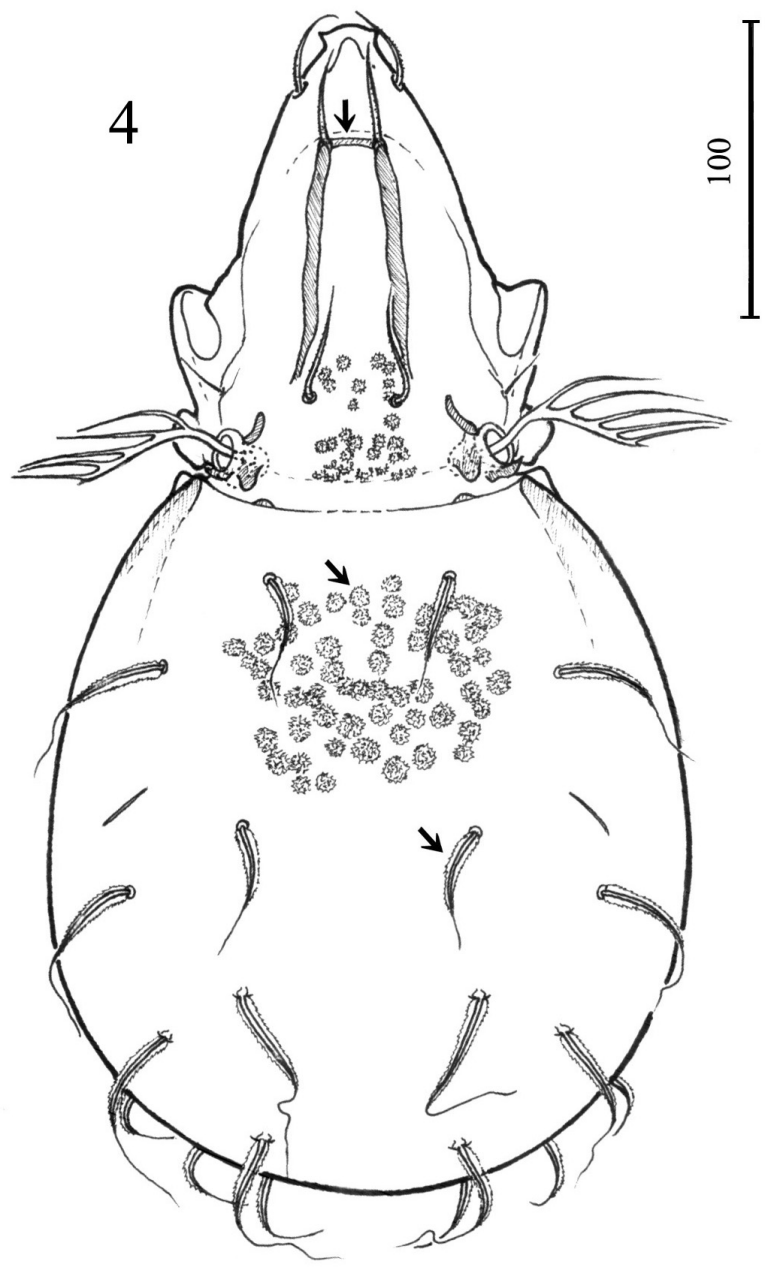

Fig. 4.- Ctenobelba (C.) parapulchellula n. sp.: Vista dorsal del cuerpo.

Fig. 4.- Ctenobelba (C.) parapulchellula n. sp.: Dorsal view of the body.

son más cortas y presenta una cóstula translamelar que une las inserciones de las setas lamelares; además el borde de los botridios presenta una pequeña expansión escamosa lateroposterior, mientras que en las bases, y hacia el interior, presentan sendos tubérculos dirigidos hacia atrás y enfrentadas a dos diminutos tubérculos del borde anterior del notogáster.

Notogáster (Fig. 4): cubierto con un cerotegumento de gránulos muy gruesos, bastante más que los del prodorso; el borde notogastral anterior es ligeramente cóncavo, con los ángulos humerales prominentes y sendos diminutos tubérculos hacia el interior; los 10 pares de largas setas notogastrales presentan la mitad basal con sendas expansiones hialinas de borde tenuemente aserrado, mientras que la mitad distal está constituida por un flagelo ondulante; las fisuras im están bien desarrolladas.

Región ventral: con un cerotegumento similar al prodorsal, presenta la región epimeral con una quetotaxia como el de la especie precedente, con las setas $1 b$ notablemente más largas que las restantes y sin los apodemas apo4 desarrollados, aunque carece del par de apófisis sejugal interior y sólo está desarrollado el par de apófisis exterior, estando reducidos los discidios a un par de pequeños tubérculos; también presenta las placas genitales con 6 pares de setas diminutas, 3 pares de setas adgenitales y las fisuras adanales iad paraanales; las patas son moniliformes, monodáctilas y desprovistas de cerotegumento.

Etimología. El epíteto "parapulchellula" hace referencia a su proximidad a la especie C. pulchellula.

Discusión. A la especie que más se asemeja es a C. pulchellula, pero la nueva especie muestra una nítida cóstula tranlamelar que la especie anterior no presenta.

\section{Ctenobelba (C.) foveolata n. sp.}

Material tiPo: Se han estudiado 12 ejemplares procedentes del Sur de España, de Córdoba, de dos muestras endogeas recolectadas por E. Banda, 11 de ellos (paratipos conservados en una preparación microscópica con Hoyer) recolectados el 22 de febrero de 1999 en Las Aljavaras, y el holotipo (conservado también en preparación microscópica con Hoyer) recolectado el 3 de noviembre de 1999 en el Arroyo de La Torre.

DESCRIPCIÓN. Dimensiones: sus medidas oscilan entre los 395-435 $\mu \mathrm{m}$ de longitud por 180-240 $\mu \mathrm{m}$ de anchura.

Prodorso (Fig. 5): en contra de lo que ocurre con las especies anteriores, en este caso no existe cerotegumento que lo cubra y sí que destaca la presencia de un marcado y prieto foveolado; el rostro presenta dos dientes romos laterales y las setas rostrales lisas; las setas lamelares, también lisas, son más largas, aproximadamente el doble que las rostrales, mientras que las interlamelares son similares a las rostrales; las cóstulas lamelares son largas y ligeramente convergentes, partiendo de sus bases y 


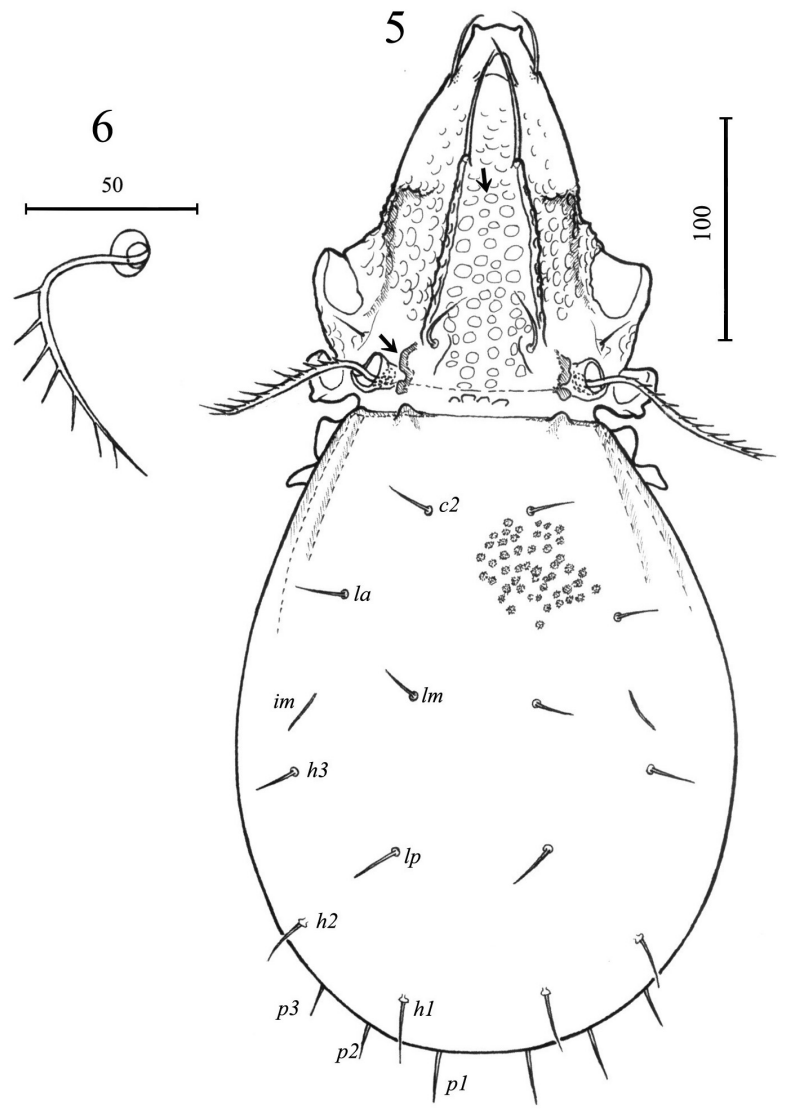

Figs. 5-6.- Ctenobelba (C.) foveolata n. sp.: 5) Vista dorsal del cuerpo; 6) sensilo. (Escala en $\mu \mathrm{m}$ ).

Figs. 5-6.- Ctenobelba (C.) foveolata n. sp.: 5) Dorsal view of the body; 6 ) sensillus. (Scale bar in $\mu \mathrm{m}$ ).

hacia atrás sendas quillas esclerotizadas que recorren el borde interno de los botridios y que acaban en sendos tubérculos enfrentados al par de apófisis medias del borde anterior del notogáster; los botridios presentan un reborde escamoso en su lado exterior, las setas exobotrídicas son cortas, finas y lisas, y los sensilos (Fig. 6), pectinados, presentan de 7 a 11 cortas ramas de longitud y separación similar.

Notogáster (Fig. 5): cubierto con un cerotegumento constituido por pequeños gránulos espiculados densa e irregularmente distribuidos, presenta el borde anterior recto, con los ángulos humerales bien marcados y con un par de apófisis medias; los 10 pares de setas notogastrales son similares, cortas, finas y lisas, y las fisuras im están bien desarrolladas.
Región ventral: la región epimeral presenta un foveolado similar al prodorsal, mientras que la placa ventral se muestra cubierta por un cerotegumento granuloso similar al del notogáster; su quetotaxia y presencia de apófisis sejugales es similar al de la especie precedente, presentando también un par de pequeños tubérculos discidiales, aunque las setas epimerales son mucho más cortas; con 6 pares de diminutas setas genitales y 3 pares de adgenitales, que son muy cortas al igual que los 2 pares de anales y 3 pares de adanales; fisuras iad paraanales y patas, al igual que las especies precedentes, moniliformes, monodáctilas y sin cerotegumento.

ETIMologíA. El epíteto "foveolata" hace referencia al nítido foveolado prodorsal que presenta.

Discusión. Se diferencia claramente de todas las demás especies del género conocidas ya que es la única que presenta el prodorso foveolado.

\section{Ctenobelba (C.) brevipilosa Mahunka, 1964}

Se trata de una especie de distribución centroeuropea (Subías, op. cit.) que fue descrita por Mahunka (1964) de Hungría. El único ejemplar estudiado, que supone primera cita para España, procede del suroeste, de una muestra endogea recolectada por J. Zaballos en Zorita (Cáceres) el 5 de marzo de 2002. De todas formas presenta algunas diferencias con la descripción original, como es el caso de una dimensiones menores: $400 \mu \mathrm{m}$ x 205 $\mu \mathrm{m}$, ya que Mahunka (op. cit.) le asigna 461-490 $\mu \mathrm{m} \times 270-343 \mu \mathrm{m}$, (aunque el mismo autor posteriormente, Mahunka, 1977, le asigna una longitud de 416-431 $\mu \mathrm{m}$ ) y un número superior de ramas en el sensilo, alrededor de 25 frente a las 12-17 que cita su autor, y que se considera podría entrar dentro de la variabilidad de la especie.

\section{Ctenobelba (C.) marcuzzii Mahunka, 1974}

Especie descrita de Italia por Mahunka (1974) fuera de donde no había vuelto a ser citada hasta ahora que lo ha sido en España. Los ejemplares estudiados proceden de dos localidades norteñas, dos de una muestra de musgo de pinar recolectada por L. S. Subías el 29 de junio de 1983 en Mambliga (Burgos), y 8 de una muestra de hojarasca de avellano recolectada por R. Outerelo el 26 de abril de 1986 en Solares (Santander). 
Ctenobelba (C.) csiszarae Mahunka, 1977

Esta cita española es la primera realizada fuera de Hungría de donde fue descrita por Mahunka (1977). Los numerosos ejemplares estudiados proceden de las siguientes muestras, todas ellas del norte de España, de Navarra: cuatro recolectados por L. S. Subías en Zudaire, en una muestra de musgo de roble recogida el 28 de junio de 1983, 16 recolectados por J. Zaballos en San Miguel de Aralar, en una muestra de suelo de rocalla recogida el 26 de enero de 1997, y otros 20 en una muestra endogea recolectada el 5 de abril de 1997 por el mismo autor y en la misma localidad.

\section{Clave de especies ibéricas de Ctenobelba s. str.}

1. Setas notogastrales con extremo flageliforme ............ 2

- Setas notogastrales sin extremo flageliforme ............. 7

2. Setas notogastrales con dilatación membranosa basal

- Setas notogastrales sin dilatación membranosa basal 6

3. Setas notogastrales y flagelo distal cortos C. (C.) parafoliata

- Setas notogastrales medianas o largas

ales liso

4. Contorno hialino de las setas notogastrales liso ................... C. (C.) foliata

- Contorno hialino de las setas notogastrales aserrado .. 5

5. Con cóstula translamelar C. (C.) parapulchellula

- Sin cóstula translamelar C. (C.) pulchellula

6. Granulado notogastral dispuesto en retículo C. (C.) mahnerti

- Granulado notogastral no dispuesto en retículo C. (C.) pseudomahnerti

7. Setas notogastrales muy cortas 8

- Setas notogastrales de logitud media 10

8. Con foveolado prodorsal C. (C.) foveolata

- Sin foveolado prodorsal 9

9. Sensilo con 9-10 ramas. Longitud de 320-354 $\mu \mathrm{m}$.... ........... C. (C.) csiszarae

- Sensilo con 12-25 ramas. Longitud de 400-490 $\mu \mathrm{m}$.. .. C. (C.) brevipilosa

10. Setas notogastrales anchas, peniciladas C. (C.) pilosella

- Setas notogastrales setiformes 11

11. Setas notogastrales barbuladas C. (C.) marcuzzii

- Setas notogastrales lisas 12

12. Sensilo con 7-10 ramas cortas .. C. (C.) apatomorpha

- Sensilo con 4-6 ramas largas 13
13. Rama basal del sensilo mucho más desarrollada y separada de las siguientes que las restantes ramas .... C. (C.) perezinigoi

- Rama basal del sensilo de longitud y separación de las siguientes como las restantes ramas C. (C.) pectinigera

\section{Agradecimientos}

Se agradece al Dr. Julio Arroyo y a los demás evaluadores sus valiosos comentarios que han contribuido a mejorar esta publicación.

\section{Referencias}

Aoki, J. I., 2007. A new species of oribatid mite of the genus Ctenobelba collected from the US Army Base on Okinawajima Island (Oribatida: Ctenobelbidae). Biological Magazine Okinawa, 45: 11-13.

Choi, S. S., 2005. A new species of the genus Ctenobelba (Acari, Oribatida) from Korea. Journal of Asia-Pacific Entomology, 8: 25-28. DOI: 10.1016/S1226-8615(08)60068-0

Iordansky, S. N., 1990. [New species and subspecies of the Oribatidae (Acariformes) from Dnepropetrovsk region.] Zoologiceskij Zurnal, 69(5): 138-142 (en ruso).

Mahunka, S., 1964. Über die Gattung Ctenobelba Balogh, 1943 (Acari: Oribatei). Opuscula Zoologica (Budapest), 5(2): 223-228.

Mahunka, S., 1974. Ctenobelba marcuzzii sp. n. eine neue Milben-Art aus Italien (Acari, Oribatida). Annales Historico-Naturales Musei Nationalis Hungarici, 66: 395-397.

Mahunka, S., 1977. Ctenobelba csiszarae sp. n. und einige Bemerkungen über die Gattung Ctenobelba Balogh, 1943 (Acari, Oribatida). Annales HistoricoNaturales Musei Nationalis Hungarici, 69: 301-304.

Pankov, A. N., 1988. [A new species C. soloduchi n. sp. of the genus Ctenobelba (Oribatei, Ctenobelbidae) from Khabarovsk Territory.] Zoologiceskij Zurnal, 67(1): 143-144 (en ruso).

Subías, L. S., 2004. Listado sistemático, sinonímico y biogeográfico de los ácaros oribátidos (Acariformes, Oribatida) del mundo (1758-2002). Graellsia, 60(Núm. extr.): 3-305. DOI: 10.3989/graellsia.2004. v60.iExtra

Subías, L. S., 2012. Listado sistemático, sinonímico y biogeográfico de los ácaros oribátidos (Acariformes, Oribatida) del mundo (excepto fósiles). http://www. ucm.es/info/zoo/Artropodos/Catalogo.pdf.

Subías, L. S. \& Shtanchaeva, U., 2012. Oribátidos ibéricos (Acari: Oribatida): Listado sistemático, incluyen- 
do nuevas citas de una familia, cuatro géneros y veinticinto especies. Revista Ibérica de Aracnología, 20: 85-103.

Weigmann, G., 2006. Hornmilben (Oribatida). Die Tierwelt Deutschlands. Begründet 1925 von Friedrich Dahl. 76 Teil. Goecke \& Evers. Keltern. $520 \mathrm{pp}$.

Recibido / Received, 2-10-2012 Aceptado / Accepted, 19-03-2013 